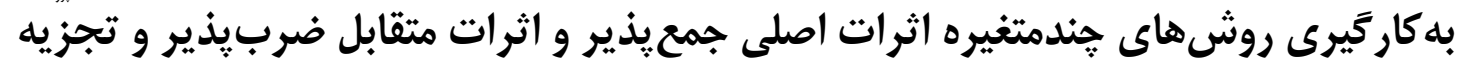

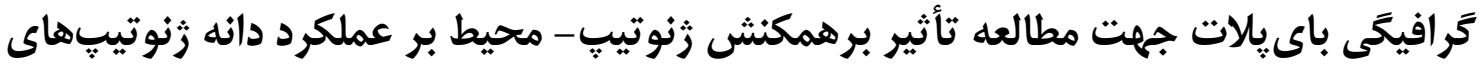

كَلرنَّ

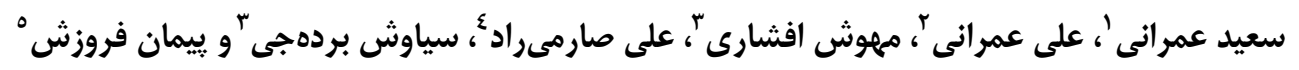

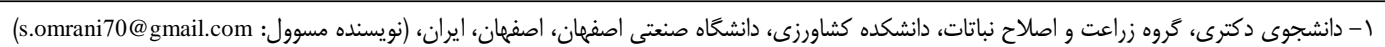

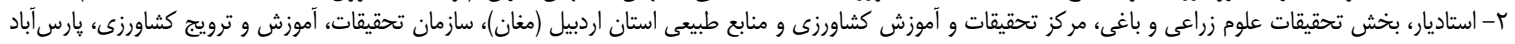

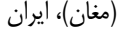

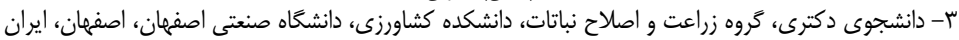

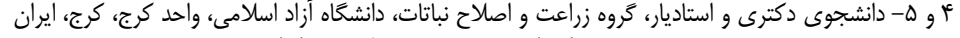

تاريخ دريافت و

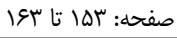

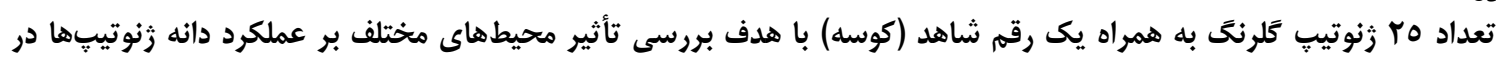

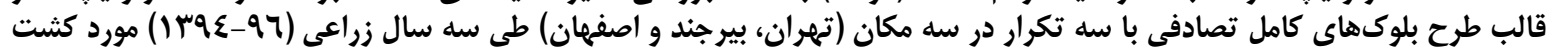

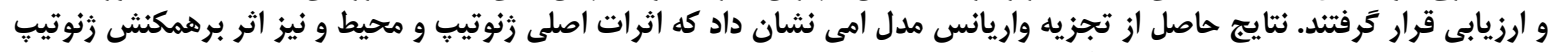

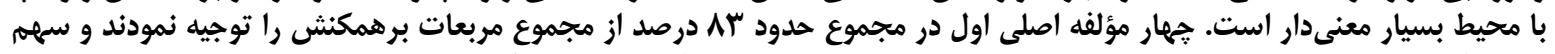

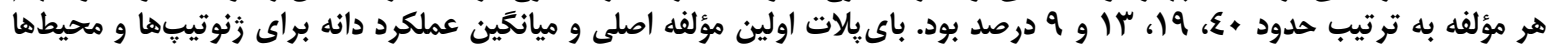

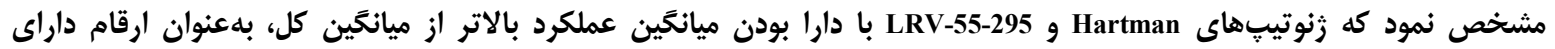

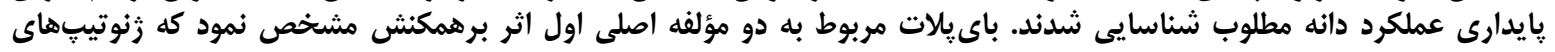

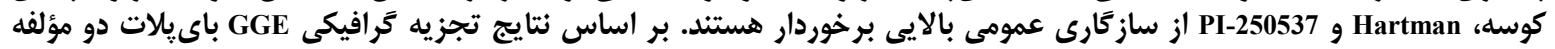

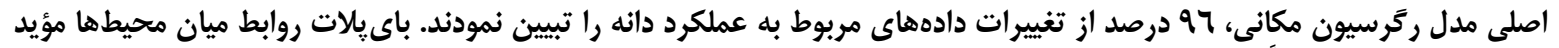

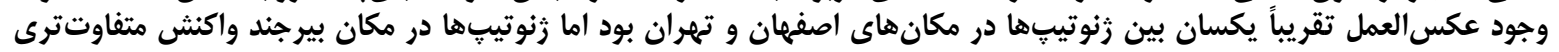

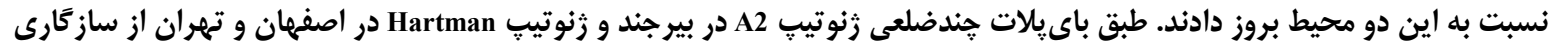

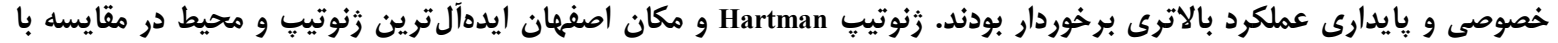

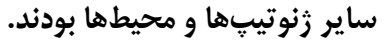

وازههاى كليدى: GGE biplot،AMMI، ابر محيط، تجزيه پايدارى، كَلرنتَ

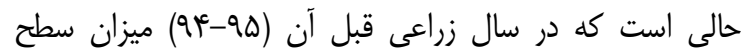

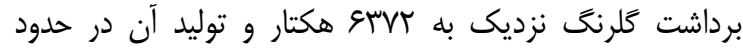

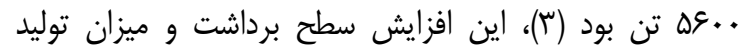

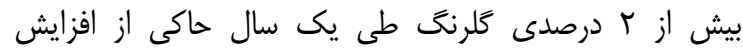

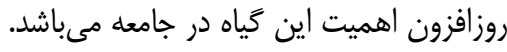

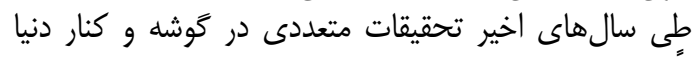

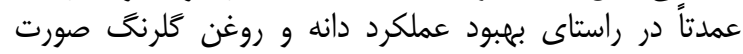

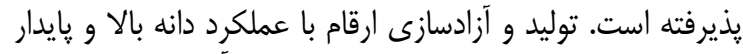

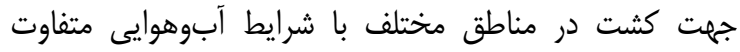

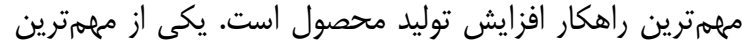

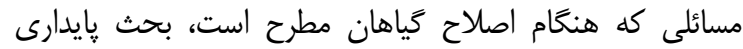

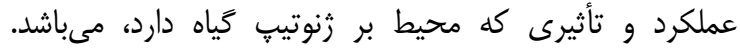

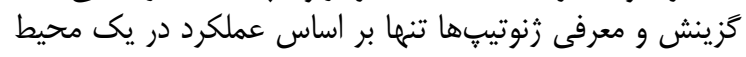

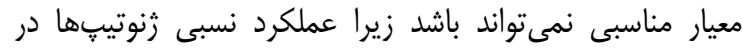

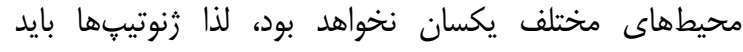
حتىالامكان در دامنه وسيع و متنوعى از شرايط محيطى لنيل
مقدمه

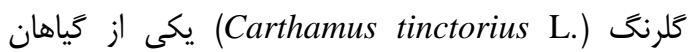

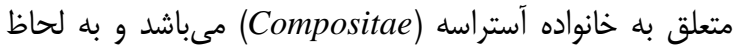

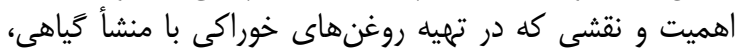

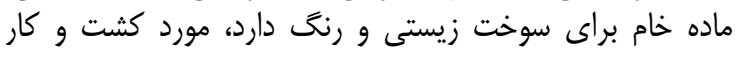

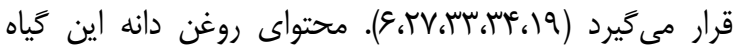

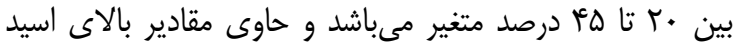

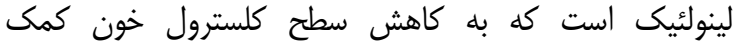

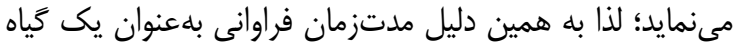

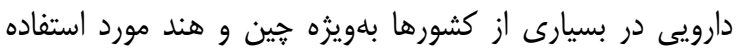

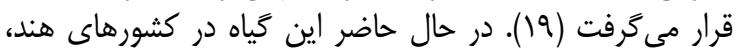

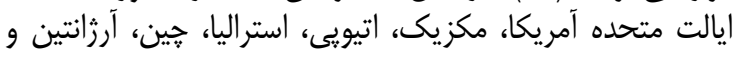

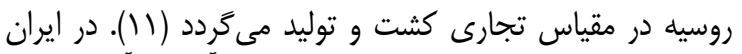

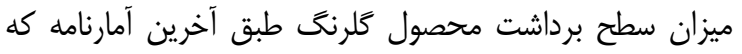

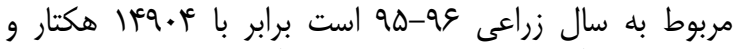

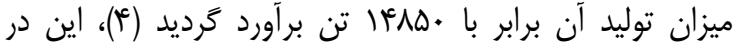




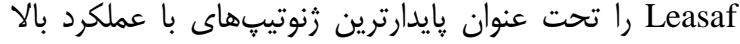

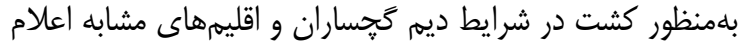

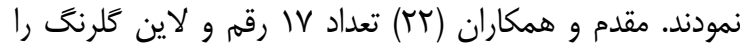

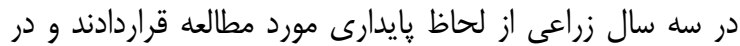

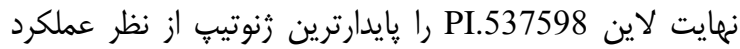

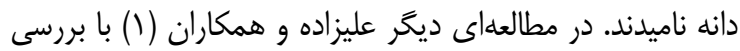

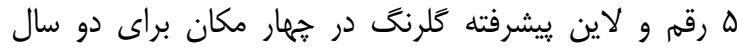

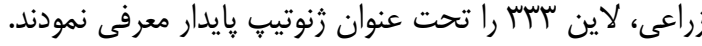

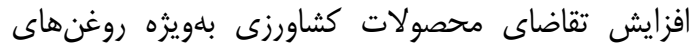

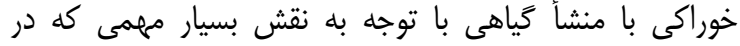

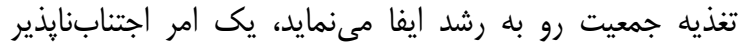

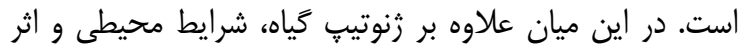

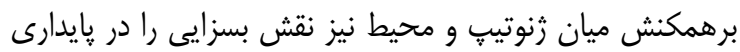

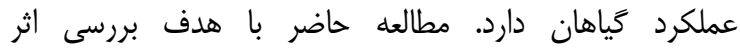

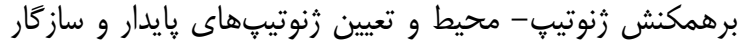

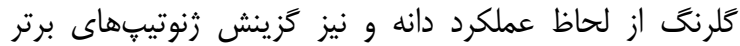

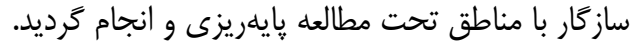

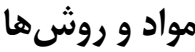

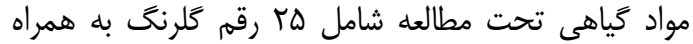

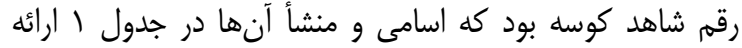

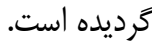

آزمايش حاضر در قالب طرح بلوكهاى كامل تصادفى با سه

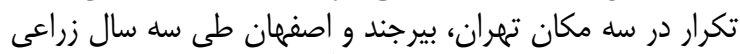

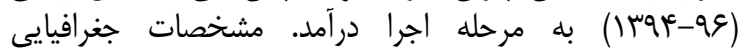

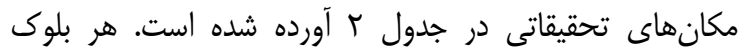

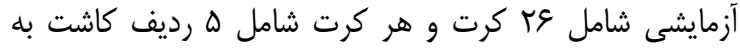

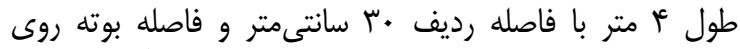

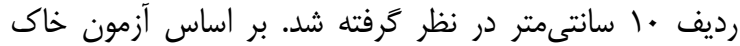

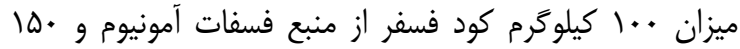

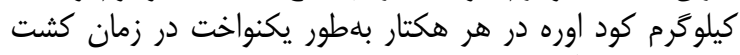

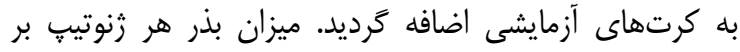

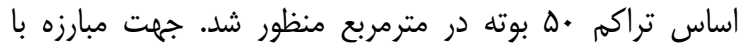

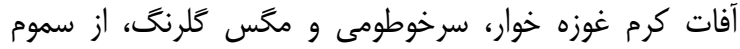

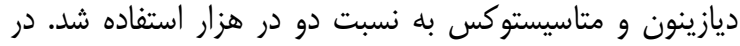

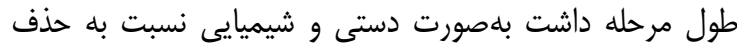

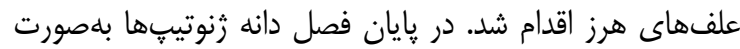

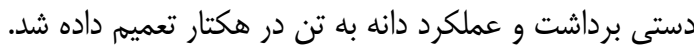

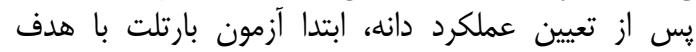

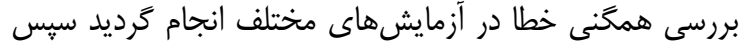

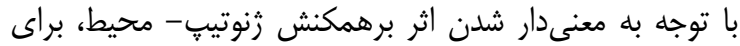

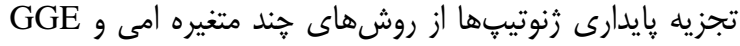
باى بلات استفاده شد. ازي نرمافزار SAS بر اساس رابطه زير بلهمنظور تجزيه يايدارى به روش امى استفاده شد:

Yger $=\mu+\alpha g+\beta \mathrm{e}+\sum \mathrm{n} \lambda \mathrm{n} \alpha \mathrm{gn} \gamma \mathrm{en}+\rho \mathrm{ge}+\varepsilon \mathrm{ge}$

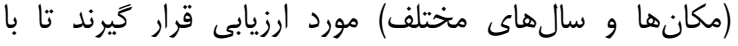

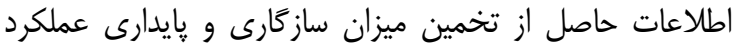

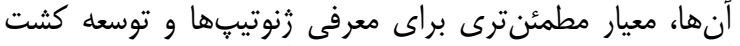

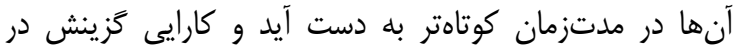

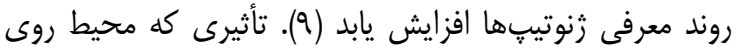

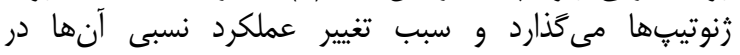

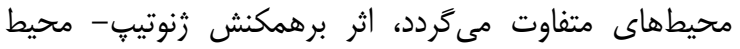

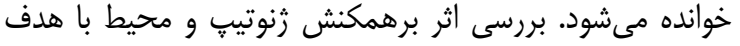

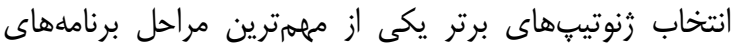

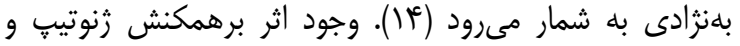

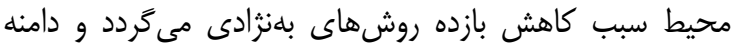

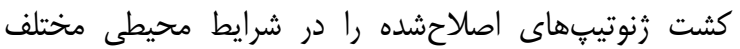

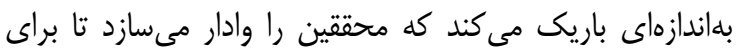

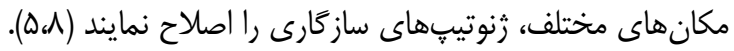

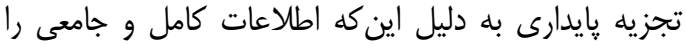

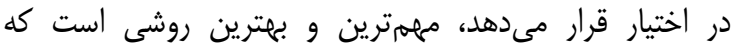

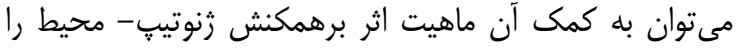

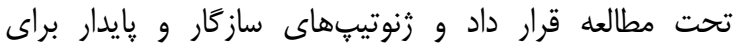

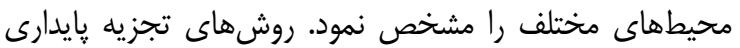

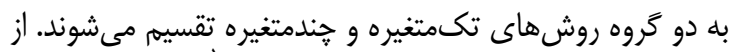

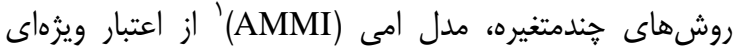

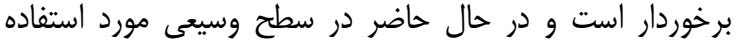

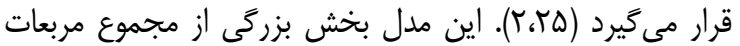

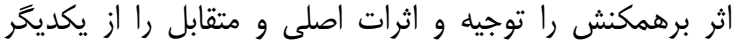

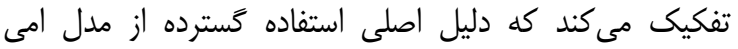

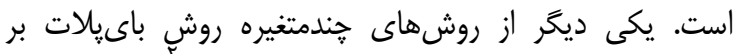

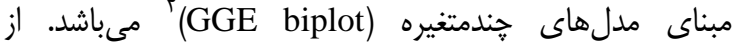

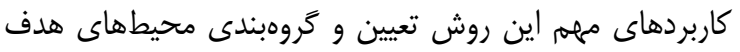

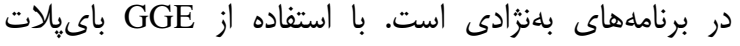

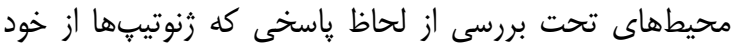

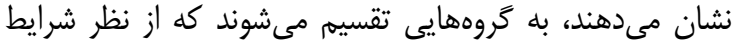

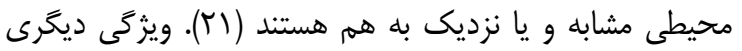

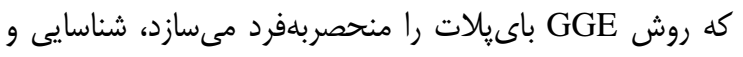

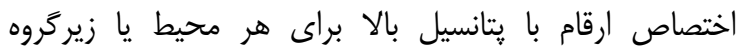

$$
\text { مىباشد (•) مجعاص (1). }
$$

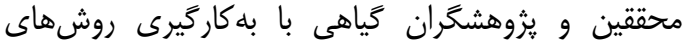

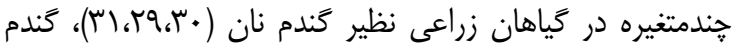

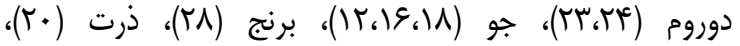

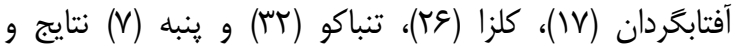

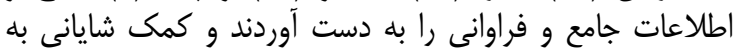

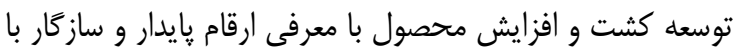

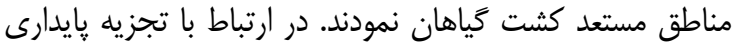

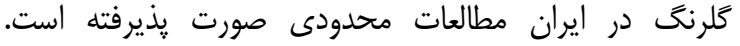

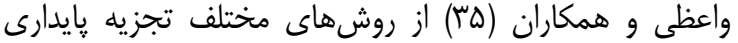

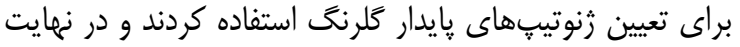

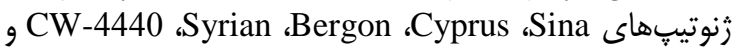


در رابطه فوق SSIPCA1 و SSIPCA2 به ترتيب مربوط به مجموع مربعات اولين و دومين مؤلفههاى اصلى برهمكنش،

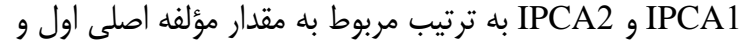

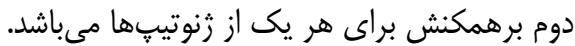

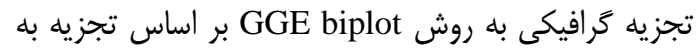
مقادير منفرد طبق رابطه زير انجام شد:

Yij $-\mu-\beta \mathrm{j}=\lambda 1 \xi \mathrm{i} 1 \eta \mathrm{j} 1+\lambda 2 \xi \mathrm{i} 2 \eta \mathrm{j} 2+\varepsilon \mathrm{ij}$

در اين رابطه Yij ميانخين زنوتيب اiم در محيط زام،

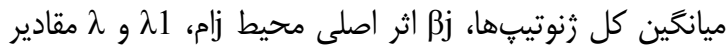

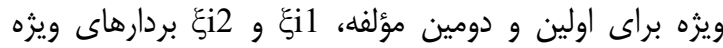

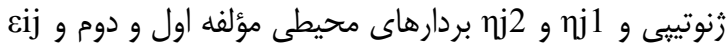
مقدار باقيمانده براى زنوتيڤ آنام در محيط زام زام هستند. جهت تجزيه دادههاى آزمايش به روش روش

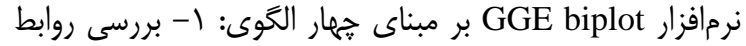

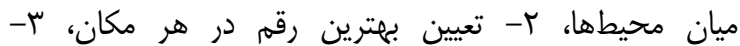

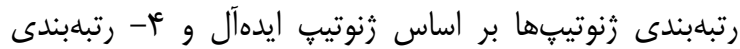
محيطها بر اساس محيط ايدهآل استفاده شد. رندائ ايدها
در اين رابطه، Yger عملكرد زنوتيب gام در محيط عام در تكرار

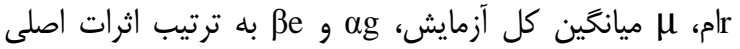

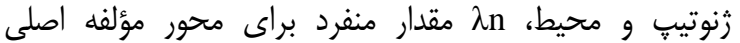

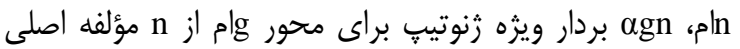

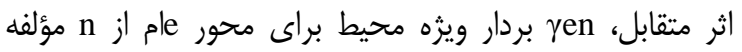

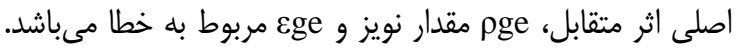

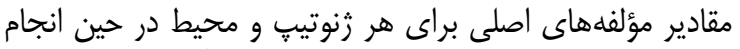

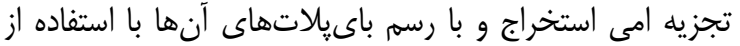

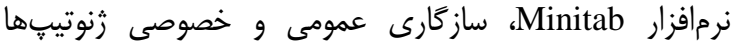
تحت بررسى قرار كرفت.

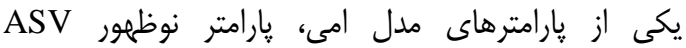

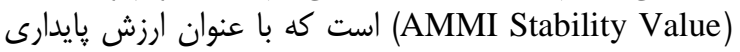

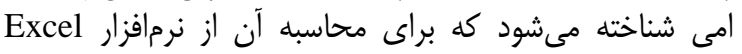
طبق رابطه زير استفاده گرديد:

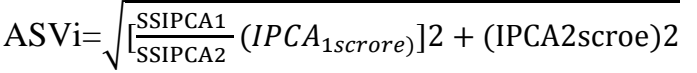

Table 1. The name and origins of studied safflower cultivar

\begin{tabular}{|c|c|c|c|c|c|c|c|}
\hline نوع زرم پيلاسم & منشأ & نام زنوتيب & شماره زنوتيٍ & نوع زرم هالاسم & منشأ & نام زنوتيتي & كد رنوتيتٍ \\
\hline رقم & آمريكا & Hartman & G14 & لاين & آمريكا & CH-5 & G1 \\
\hline لاين & ايران & C111 & G15 & لاين & آمريكا & CW-74 & G2 \\
\hline لاين & ايران & S149 & G16 & لاين & ֶِاكستان & PI-250190 & G3 \\
\hline رقم بومى & ايران & Zarghan279 & G17 & لاين & جين & PI-506426 & G4 \\
\hline لاين & ايران & M420 & G18 & لاين & مكزيك & $22-191$ & G5 \\
\hline لاين & آمريكا & CW-4440 & G19 & لاين & ايران & A2 & G6 \\
\hline لاين & فلسطين & PI-253384 & G20 & رقم & آمريكا & Gila & G7 \\
\hline لاين & ايران & E2428 & G21 & لاين & ايران & E2417 & G8 \\
\hline لاين & ايران & LRV-51-51 & G22 & لاين & ايران & $\mathrm{C} 121$ & G9 \\
\hline لاين & يرتنال & PI-258417 & G23 & رقم بومى & سوريه & Syrian & G10 \\
\hline لاين & مصر & PI-250537 & G24 & لاين & ايران & LRV-55-295 & G11 \\
\hline لاين & تر كيه & 301055 & G25 & لاين & مكزيى & Kino-76 & G12 \\
\hline رقم بومى & ايران & كوسه & G26 & لاين & آلمان & 62918 & G13 \\
\hline
\end{tabular}

Table 2. Geographical characteristics of the experimental locations

جدول ا- نام و منشأ ارقام كلرنگ تحت مطالعه

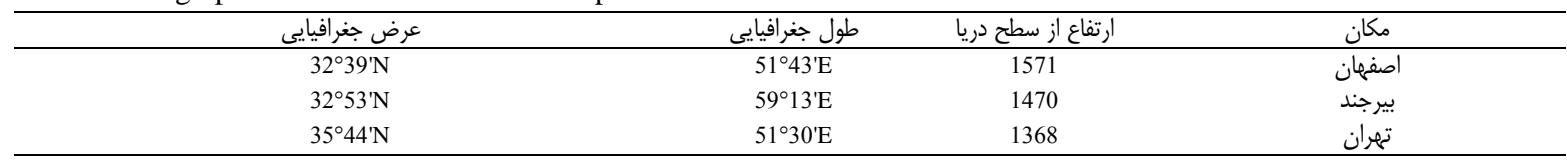

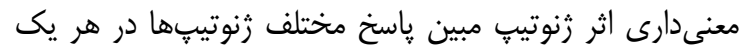

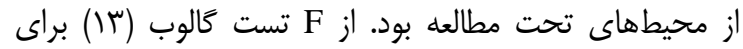

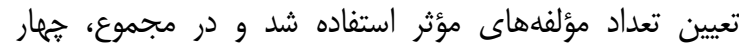

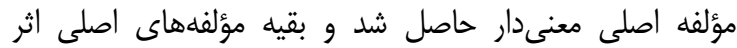

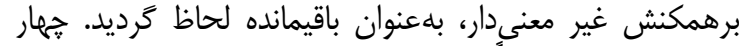

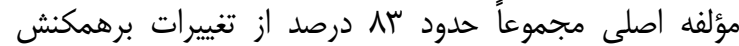

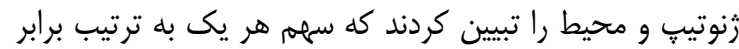

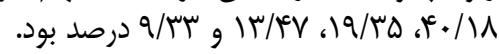

\section{نتايج و بحث}

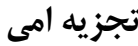
نتايج حاصل از تجزيه واريانس امى در جدول س ارائه شده

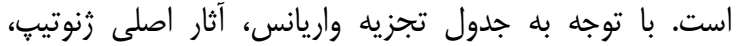

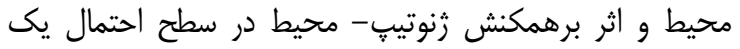

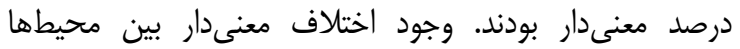

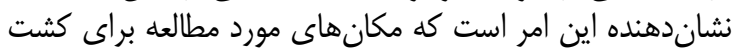

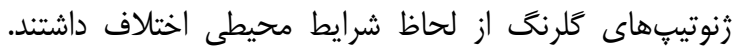




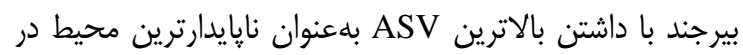

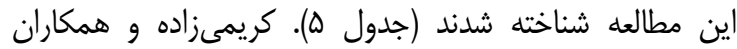

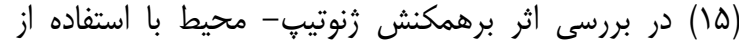

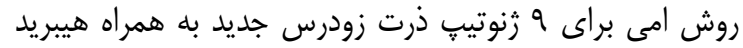

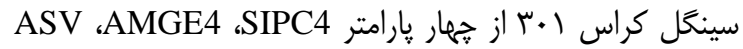

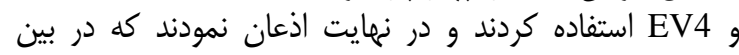
ڤارامترهاى مدل امى نتايج حاصل از پارامتر صحت بالايى برخوردار است.

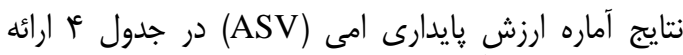

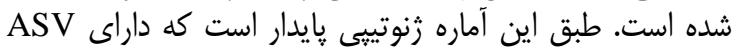

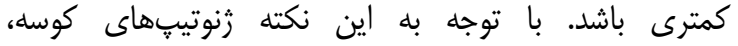

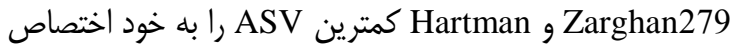

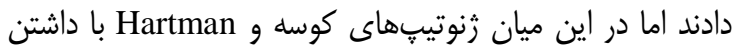

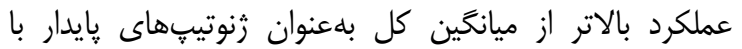

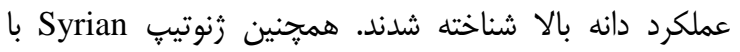

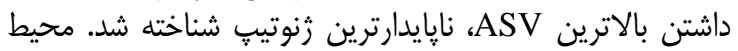

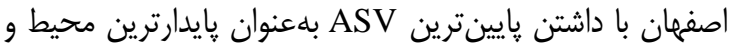

جدول س- تجزيه واريانس امى براى عملكرد دانه زنوتيڤهاى گلرنخ (سه مكان×سه سال) Table 3. Analysis of variance of AMMI model for grain yield of safflower genotypes (3 locations $\times 3$ years)

\begin{tabular}{|c|c|c|c|c|}
\hline واريانس توجيه شده (\%) & ميانگَين مربعات & مجموع مربعات & درجه آزادى & منابع تغييرات \\
\hline.$/ \mu T$ & .1 .94 &.$/ 1 T \Delta$ & $r$ & بلوك \\
\hline Q/DT & . & $r / 11$. & $\wedge$ & محيط \\
\hline$\Delta q / F$. & $\cdot / 9 \cdot 0$ & Tr/GT & $r \Delta$ & رُنوتيب \\
\hline $18 / 94$ & . & $q / \& \Delta$ & $r .$. & رنوتيبِ×حيط \\
\hline $4 . / 19$ & $\cdot / \cdot 11$ & $r / \Delta Q T$ & rr & IPCA1 \\
\hline $19 / \% \omega$ & $\cdot|\cdot+4|$ & $1 / T<q$ & r. & IPCA2 \\
\hline $\mid r / F V$ &.$/ .41$ &.$/ 189$ & rᄉ & IPCA3 \\
\hline س & " שr./. &.$/ 9 \cdot r$ & re & IPCA4 \\
\hline $\mid V / \& \Delta$ & $. / \cdot \Delta)^{\mathrm{ns}}$ & ג & Af & باقيمانده (نويز) \\
\hline $\mathrm{IV} / \mathrm{VA}$ &.$/ .14$ & $s / V V^{6}$ & 199 & خطا \\
\hline \multicolumn{3}{|c|}{$9 / 94$} & & ضر يب تغييرات (CV\%) \\
\hline
\end{tabular}

Table 4. Mean grain yield and principle component of safflower genotypes

\begin{tabular}{|c|c|c|c|c|c|c|}
\hline ASV & IPCA3 & IPCA2 & IPCA1 & ميانگين عملكرد (تن در هكتار) & شماره زنوتيّ & شماره رنوتيب \\
\hline.$/ 09$ &.- .1 .49 & $-\cdot \cdot / \mu \cdot \mu$ &.$- / T A F^{F}$ & $1 / \pi \omega$ & $\mathrm{CH}-5$ & G1 \\
\hline.$/ \mu f$ &.$- / .9$. &.$/ 149$ &.$- / 1 Q V$ & 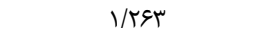 & CW-74 & G2 \\
\hline .10 . & . / N & Tr & - . & $1 / 4 / 9$ & PI-250190 & G3 \\
\hline.$/ 19$ &.$/ \cdot \Delta \Lambda$ & س ( - (1./. & .1 .94 &.$/ 999$ & PI-506426 & G4 \\
\hline . &.$- / 190$ &.$/ 1 \Lambda$ & $.1 .9 \mathrm{~V}$ & $1 / 11 \mathrm{~V}$ & 22-191 & G5 \\
\hline$\cdot / \kappa \Delta$ & . &.$- / \mid \mathrm{VI}$ & .1 .99 & $1 / 4 \mathrm{VA}$ & $\mathrm{A} 2$ & G6 \\
\hline .19 &.$- / \cdot \Delta \Delta$ & $\cdot 1 \cdot v^{k}$ & $.1 \cdot \mathrm{VQ}$ & Tr// & Gila & G7 \\
\hline . $/ \mu$. &.$/ 19 \Delta$ &.$/$ rar & -.1 .49 & $1 / 491$ & E2417 & G8 \\
\hline.$/ 4$. & 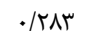 & 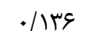 &.$- / 194$ & תr & $\mathrm{C} 121$ & G9 \\
\hline I/R &.$/ . .1$ &.$- / 191$ & سזצ/. & 1/R. & Syrian & G10 \\
\hline.$/ 19$ &.$/ M F$ &.$- / I D F$ &.$|\cdot r|$ & I/FAV & LRV-55-295 & G11 \\
\hline ع &.$/ . \mu F$ &.$- / 1 r q$ &.$/ 118$ & $1 / \Gamma \Delta V$ & Kino-76 & G12 \\
\hline$\cdot / \pi$ & -||$|r|$ & سז.|. & $-.11 \cdot 9$ & $1 / k^{4}$ & 62918 & G13 \\
\hline . & . Tyeg & $-.1 .9 \mathrm{~V}$ & -.1 .149 & V/DFQ & Hartman & G14 \\
\hline . &.$- / 199$ &.$/ \Delta \Delta \Lambda$ & $-\cdot / 1 \cdot 1$ & 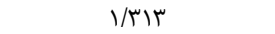 & C111 & G15 \\
\hline$\cdot / r \Delta$ &.$/ 118$ & س &.$- / 1 r q$ & $1 / \pi \cdot 9$ & S149 & G16 \\
\hline.$/ 11$ &.$- / / V \wedge$ & $-.11 \cdot 9$ &.$- / \cdot 1$ & . ANes & Zarghan279 & G17 \\
\hline . & /. Th & 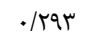 & 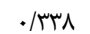 & $1 / 4 \cdot r$ & M420 & G18 \\
\hline פq/א. &.$- / 198$ & $\cdot \mid \cdot 11$ &.$/ 14 q$ & $1 / 1+\wedge$ & CW-4440 & G19 \\
\hline . &.$/ 119$ & $-\cdot / r \cdot 9$ & r.人/. & . & PI-253384 & G20 \\
\hline.$/ M F$ & $.1 . .9$ &.$\mu r$ &.$/ M F$ & I/אET & E2428 & $\mathrm{G} 21$ \\
\hline.$/ T V$ & $.1 . .9$ & . Igr &.$- / 1111$ & $1 / .99$ & LRV-51-51 & $\mathrm{G} 22$ \\
\hline عس/. &.$- / 1 F a$ & - - /YYs & $-\cdot / 1 \Lambda$ & $1 / 11$. & PI-258417 & $\mathrm{G} 23$ \\
\hline.$/ 11$ & $-\cdot / \cdot r$. &.$/ \cdot 1$ & .1 .91 & $1 /$ TFq & PI- 250537 & $\mathrm{G} 24$ \\
\hline . Th &.$- / \% 19$ &.$- / .94$ & $-\cdot|r|$. & . /ArF & 301055 & G25 \\
\hline .1 .9 & $-\cdot / 1 \cdot v$ &.$- / \cdot r$. & $-.1 .4 f^{2}$ & $1 / T / \Lambda$ & كوسه & G26 \\
\hline
\end{tabular}


Table 5. The mean grain yield and the principle component for studied environments

\begin{tabular}{|c|c|c|c|c|c|c|c|}
\hline ASV & IPCA3 & IPCA2 & IPCA1 & 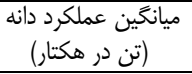 & سال & نام مكان & كد مكان \\
\hline $1 / 1 \%$ & $\cdot / / \Delta Q$ & $\cdot / \cdot \wedge \Delta$ & $\cdot|\Delta V|$ & $1 / 199$ & 1494 & بيرجند & BIR-Y1 \\
\hline$\cdot / \Lambda$ & $-\cdot / 1 f$. & /.48 & $\cdot / 4 \cdot 9$ & 1/Tre & $1 \% 90$ & بيرجند & BIR-Y2 \\
\hline سو/. &.$- / I V t$ & - &.$/ 419$ & l/IVe & عqזו & بيرجند & BIR-Y3 \\
\hline . /MF & -.1 .9$. & - . & $-.1 .4 q$ & $1 / 190$ & Irq4 & اصفهان & ESF-Y1 \\
\hline 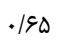 & $-\cdot / r \cdot 9$ &.$- / F \uparrow \Lambda$ &.$- / \mu t$. & $1 / 7 / 9$ & $1 \% 90$ & اصفهان & ESF-Y2 \\
\hline 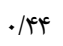 & . & $-\cdot / T V T$ & $-.|| n \mid$ & I/IVQ & & اصفهان & ESF-Y3 \\
\hline$\cdot \mid \Delta \Lambda$ & $\cdot / \cdot M$ & $\cdot / 4 \cdot 9$ & $-\cdot / r \Delta \cdot$ & I/TYA & Irq4 & تهران & THE-Y1 \\
\hline$\cdot / V 1$ & - & .1 .91 & $-\cdot / \mu \Delta Q$ & $1 / T V V$ & $1 \% 90$ & تهران & THE-Y2 \\
\hline .194 & $\cdot|\cdot v|$ &.$/ F \Delta \Lambda$ & • & וזו/ו & Iras & تهران & THE-Y3 \\
\hline
\end{tabular}

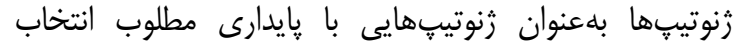

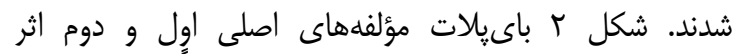

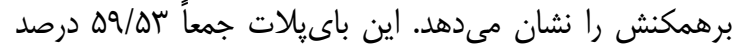

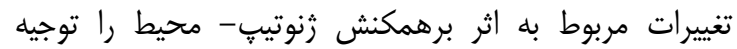

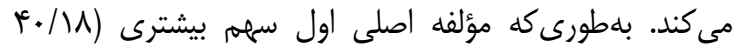

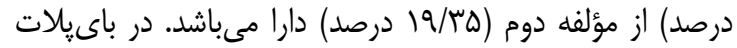

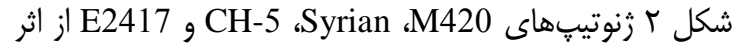
برهمكنش بسيار بزركى برخوردار بودها و وبه همين دليل نايايدار

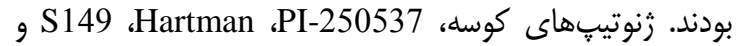

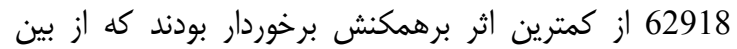

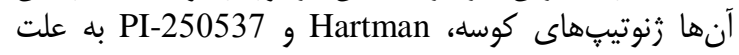

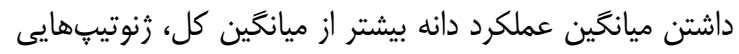

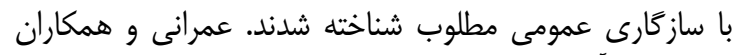

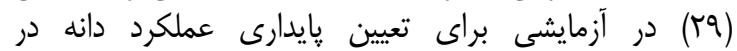

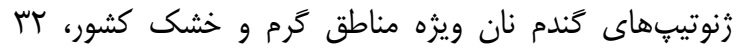

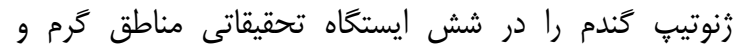

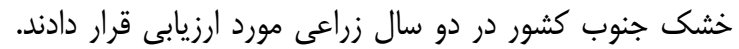

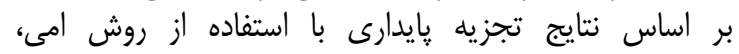

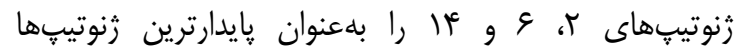
شناسايى و معرفى كردند.
شكل ا باىيلات ميانكين عملكرد دانه زنوتيِّها و اولين

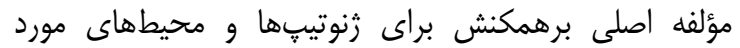

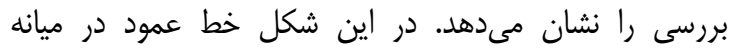

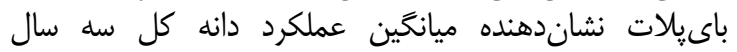

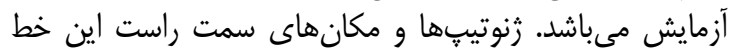

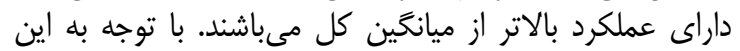

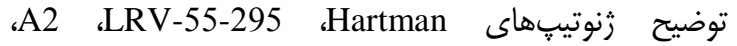

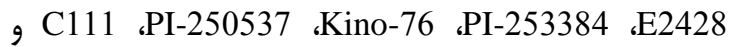

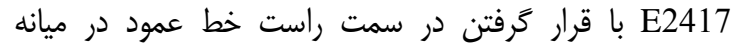

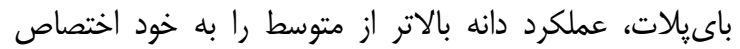

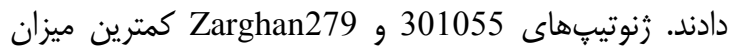

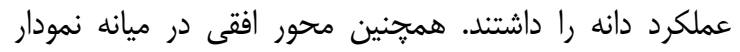

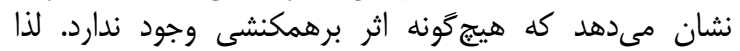

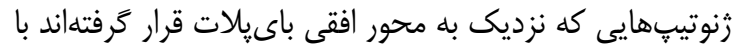

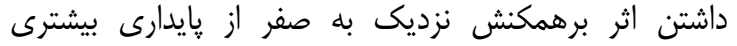

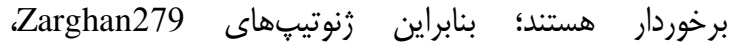

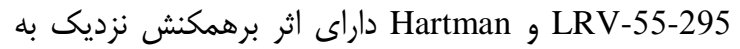

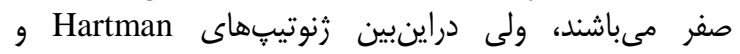

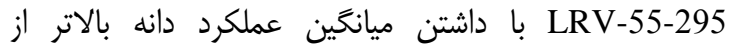
ميانكين كل و كمترين ميزان اثر برهمكنش نسبت دانئ به ساير الاتر 


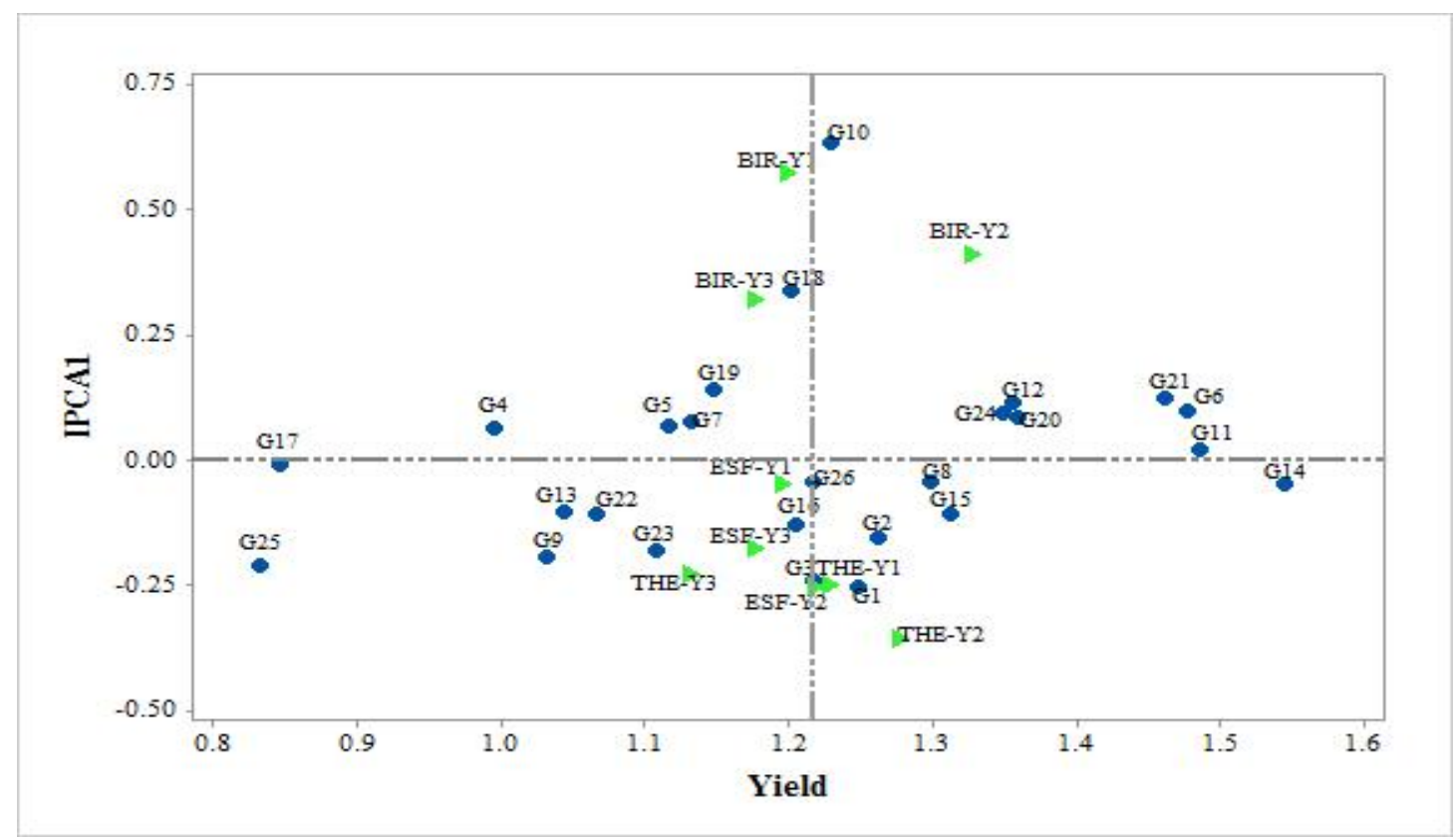

شكل 1- باى يلات ميانكين رنوتيِها و اولين مؤلفه اصلى براى زنوتيِها و مكانهاى موردبررسى

Figure1. Mean yield biplot of genotypes for studied locations with first their principle component

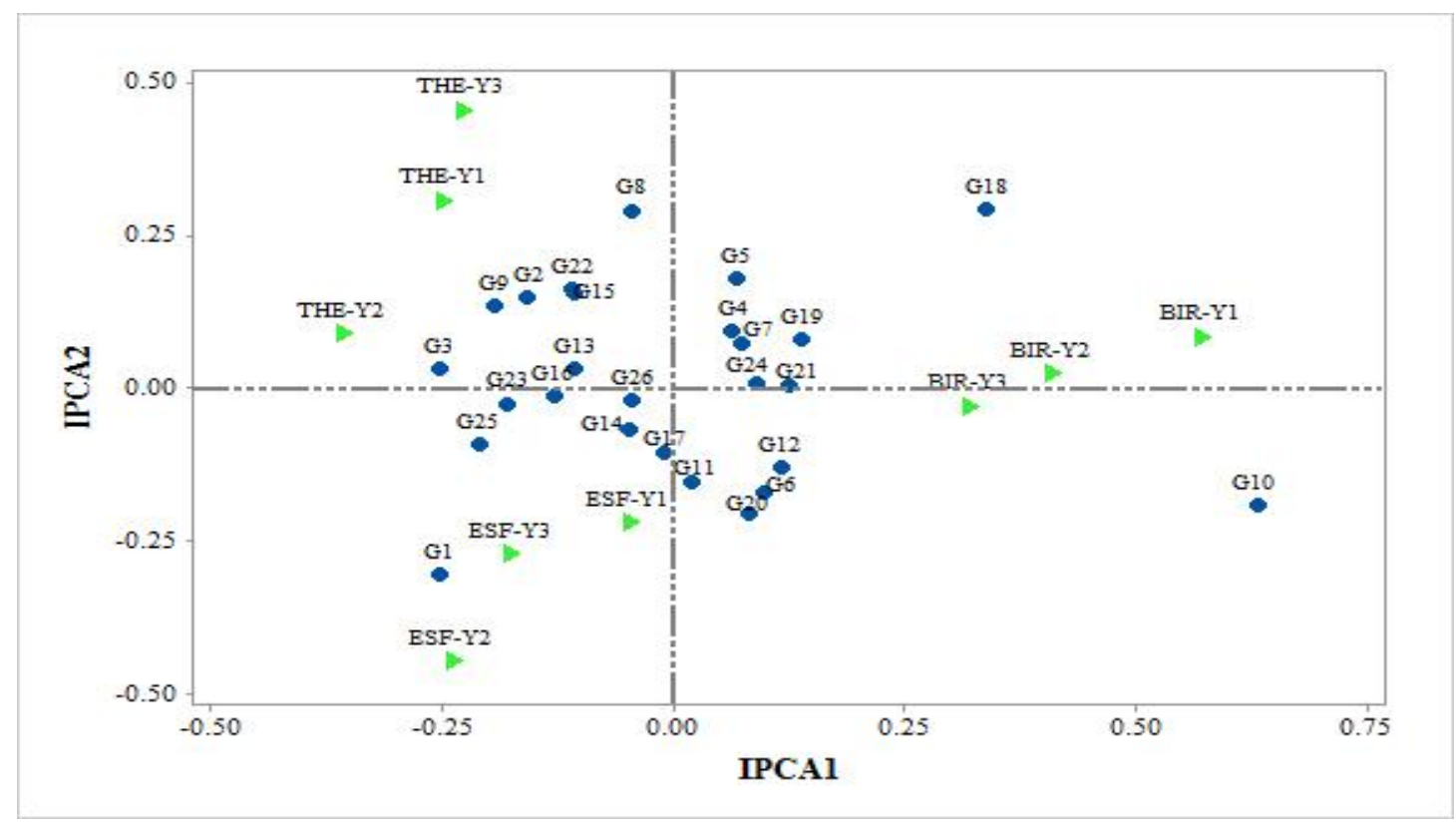

شكل r- باىٍات دو مؤلفه اصلى اول و دوم براى زنوتيِها و مكانهاى موردبر رسى

Figure 2. Bipolt of the first and second principle components for studied genotypes and different locations 
كه زنوتيب A2 بهترين زنوتيب براى منطقه بيرجند است.

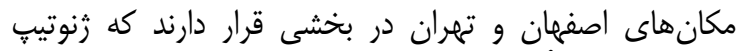
Hartman

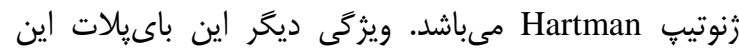

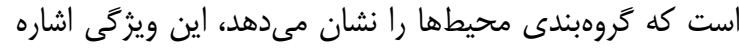

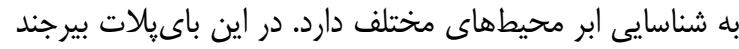

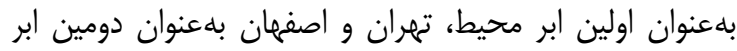

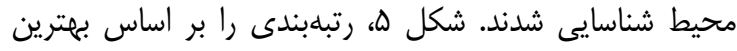

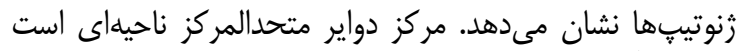

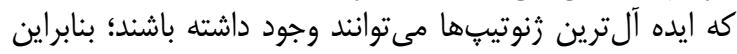

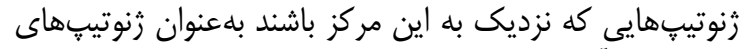

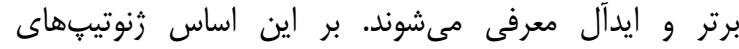
Hartman ايدهآلترين و زنوتيبهاى

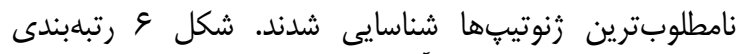

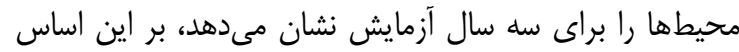

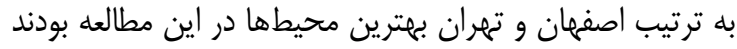

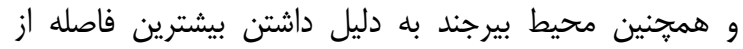

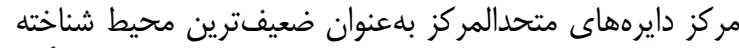

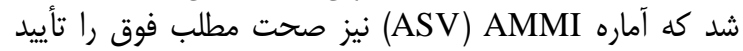

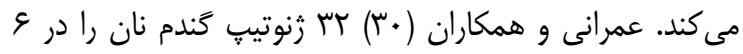

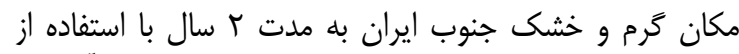

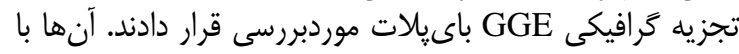

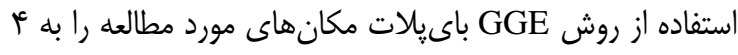

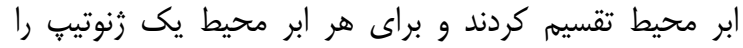
بلهنوان زنوتيب برتر انتخاب كردند.

\section{تجزيه ترافيكى GGE باى يلات}

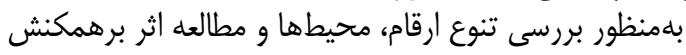

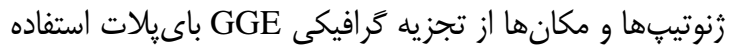

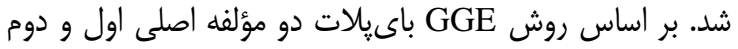
عو درصد از (AXIS2=PC2= و و AXIS1=PC1=人V/ $/$ )

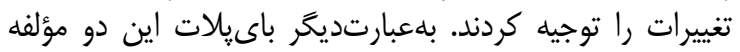

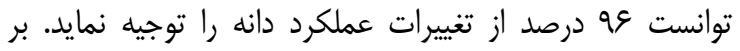

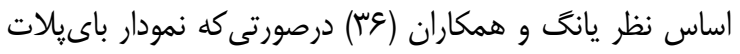

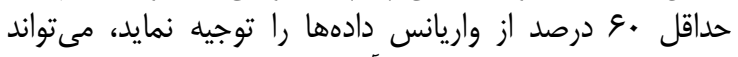

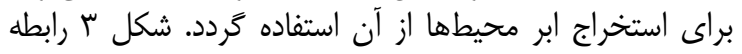

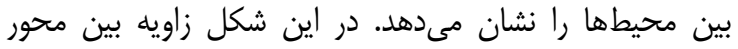

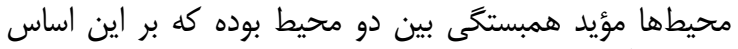

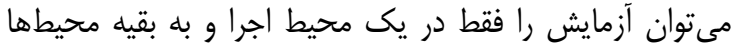

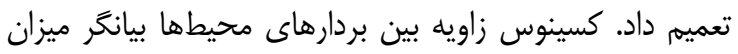

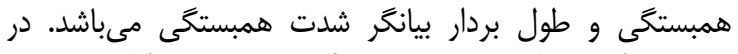

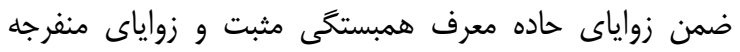

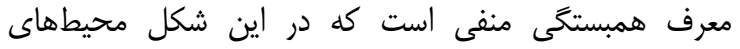

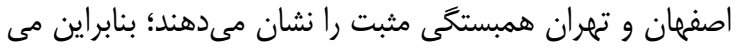

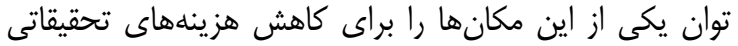

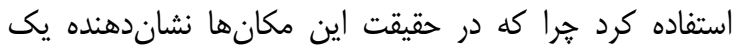

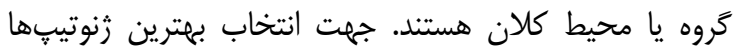

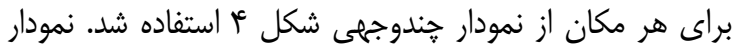

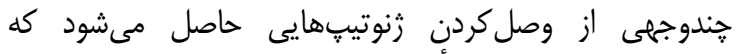

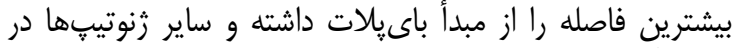

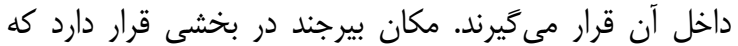
زنوتيٍ A2 در رأس آن قرار گرفته است و اين بدين معناست قرار دارد

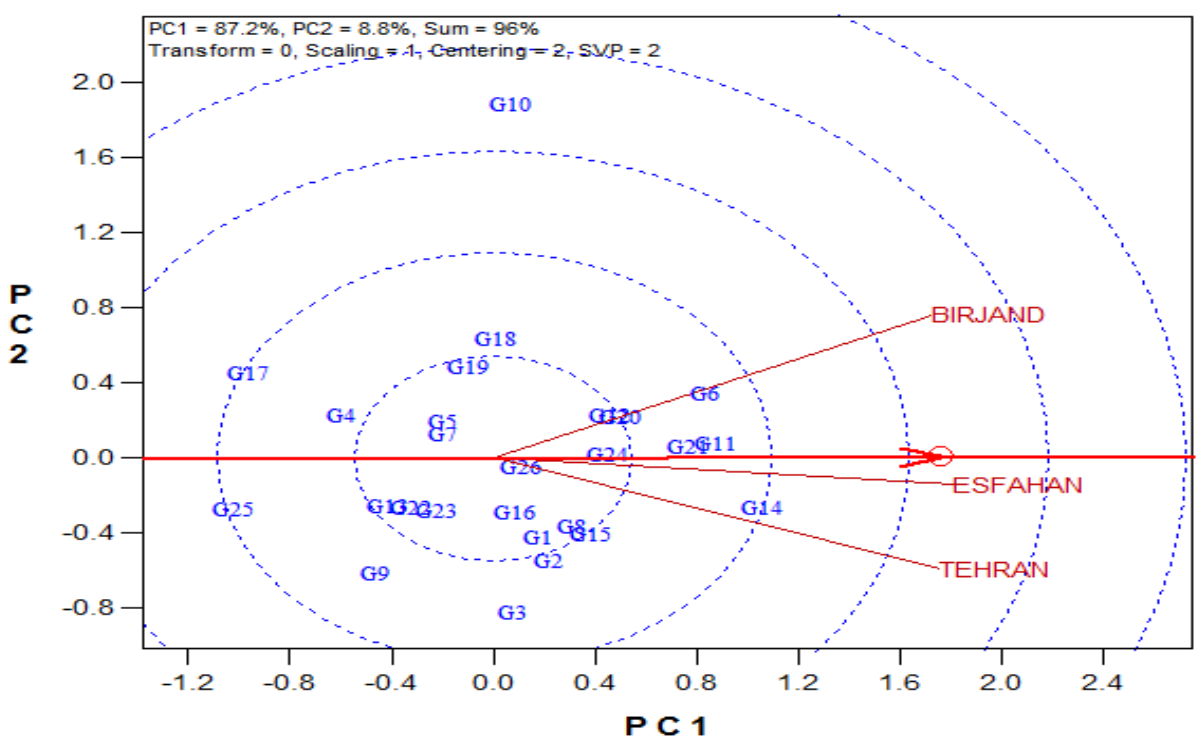

شكل س- نمودار بررسى روابط بين محيطها با استفاده از روش GGE Biplot

Figure 3. Diagram of correlations among environments using the GGE biplot method 


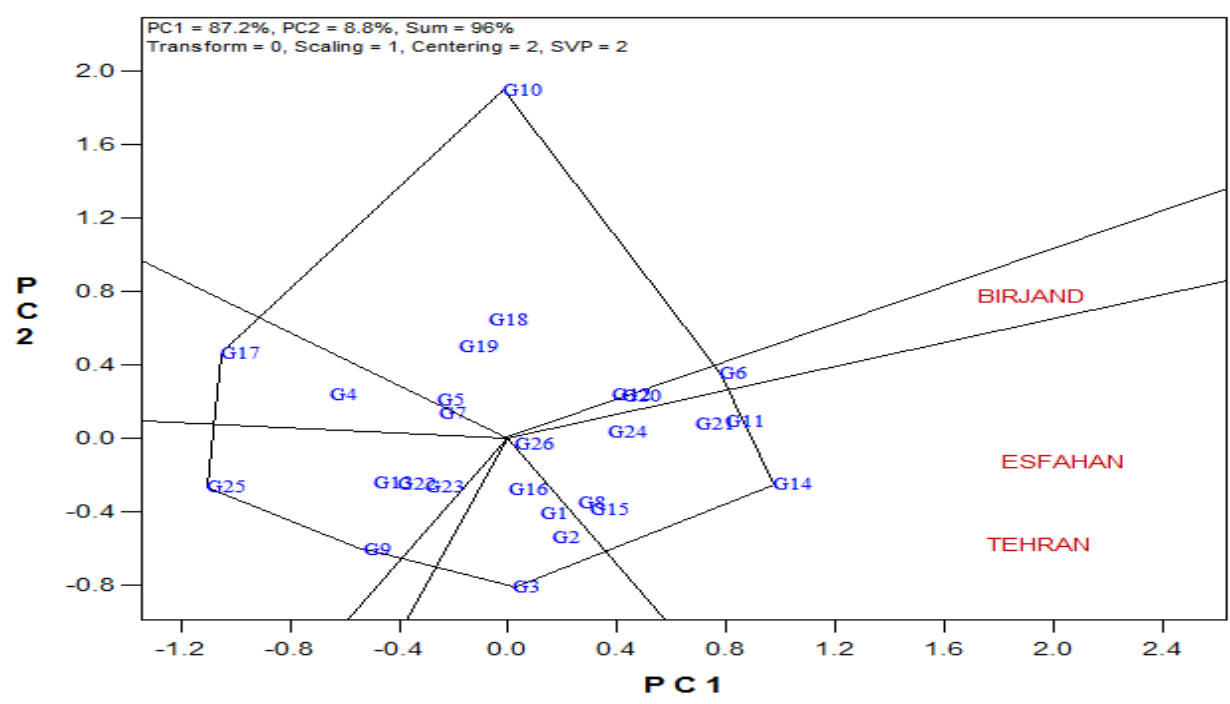

شكل זٔ- نمودار קندوجهى GGE Biplot كه نشاندهنده پر محصولترين زنوتيبها در هر محيط است

Figure 4. Polygons of GGE biplot method that display the most productive genotypes in each environment

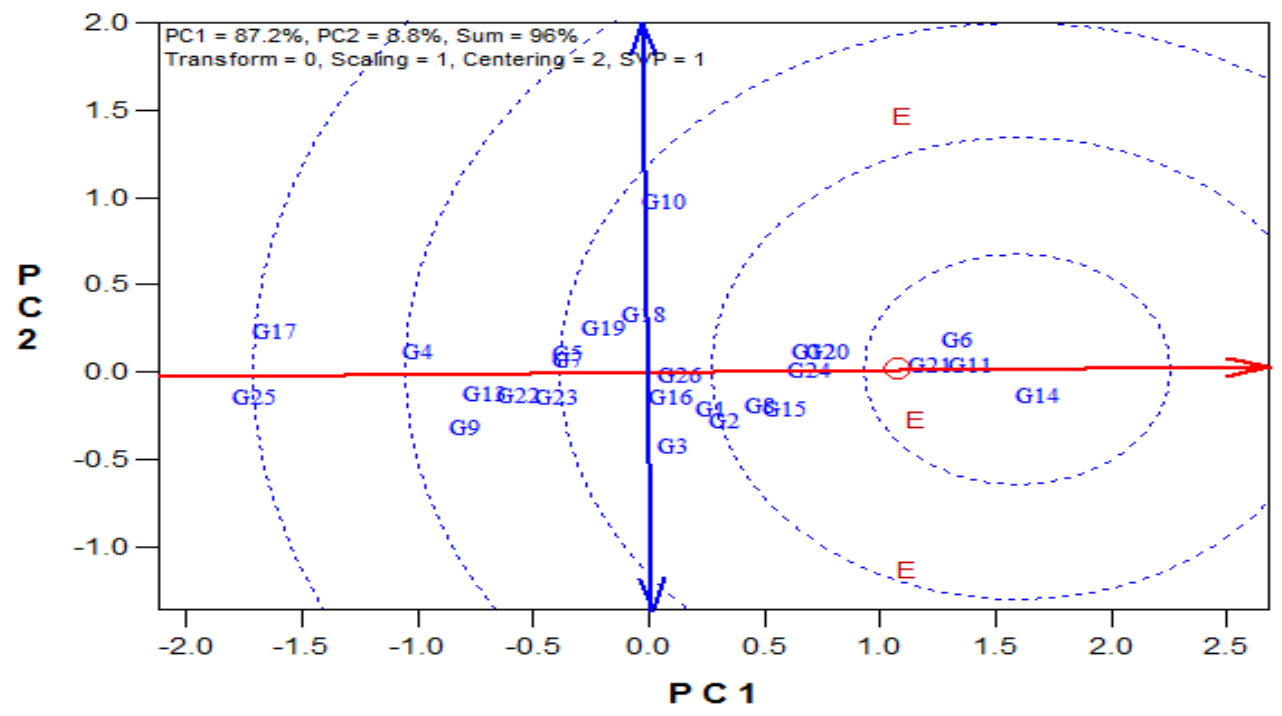

شكل ه- نمودار زنوتيب ايدهآل فرضى براى ارزيابى زنوتيڤها در سه سال آزمايش

Figure 5. Chart of Ideal genotype to evaluate genotypes in three years 


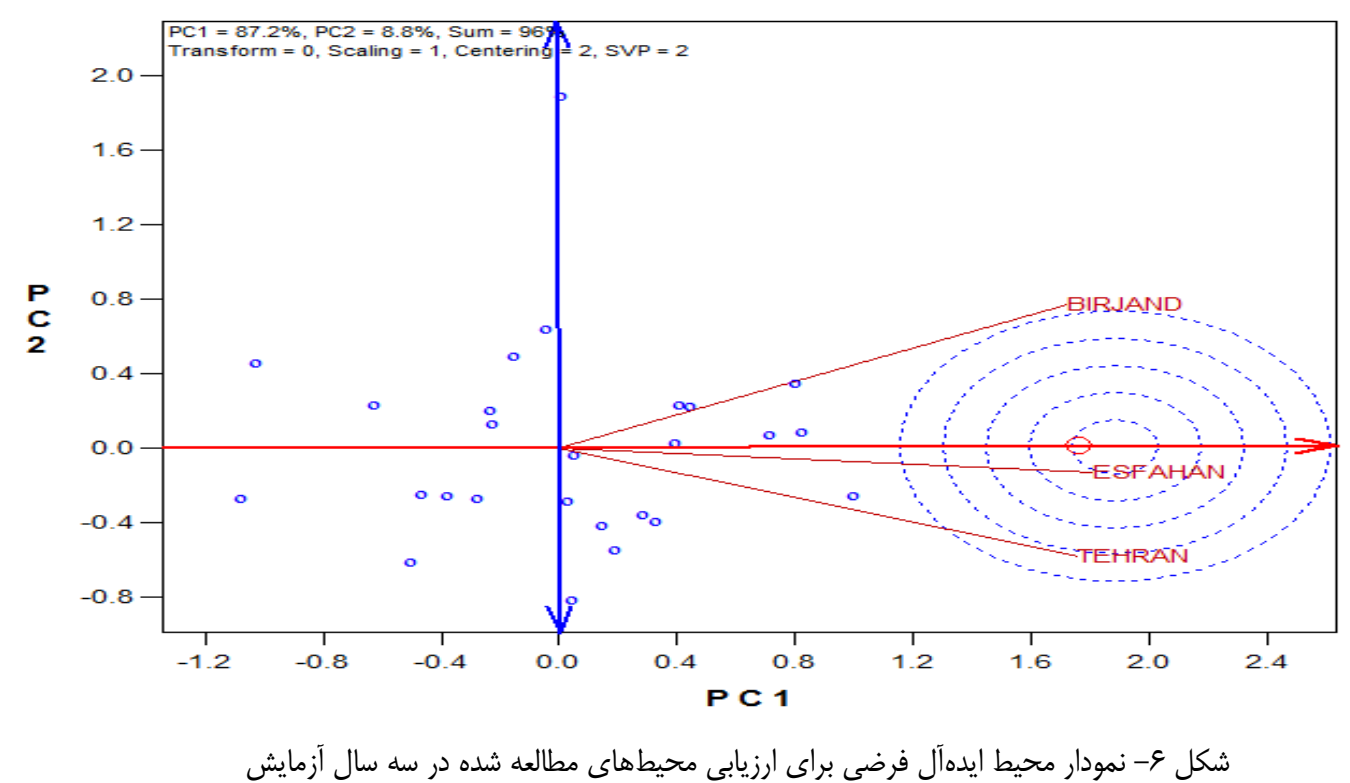

Figure 6. Chart hypothetical ideal environment for three years studied environmental assessment

\section{منابع}

1. Alizadeh, K., M. Eskandari, A. Shariati and M. Eskandari. 2008. Study on spring type safflower lines suitable for cold drylands using GGE biplots. World Journal of Agricultural Sciences, 4(6): 726-730.

2. Annicchiarico, P., L. Russi, E. Piano and F. Veronesi. 2006. Cultivar adaptation across Italian locations in four turfgrass species. Crop Science, 46: 264-272.

3. Anonymous. 2017. Agriculture-Iran-Statistics.https: //www.maj.ir /Dorsapax /userfiles /Sub65 / Amarnamehj 194-95-site.pdf.

4. Anonymous. 2018. Agriculture-Iran-Statistics.https://www.maj.ir/Dorsapax/userfiles/Sub65/Amarnamehj19596-site.pdf.

5. Becker, H.B. and J. Leon. 1988. Stability analysis in plant breeding. Plant Breeding, 101: 1-23.

6. Bergman, J.W. and R.F. Charles. 2008. Evaluation of safflower and other oil seed crops grown in the United States Northern Plains region for biofuels/ biobased products. In: Proceedings of VIIth International Safflower Conference, 3-6 November, Wagga Wagga, Australia.

7. Campbell, B.T. and M. A. Jones. 2005. Assessment of cultivar x environment interactions for yield and fiber quality in cotton performance trials. Euphytica, 144: 69-78.

8. Crossa, J., H.G. Gauch and R.W. Zobel. 1990. Additive main effects and multiplicative interaction analysis of two international maiz cultivar trials. Crop Science, 30: 493-500.

9. Farshadfar, E. 1998. Application of Biometrical Genetics in Plant Breeding. Secend edition. Razi University Publications. Kermanshah, Iran, 396 pp (In Persian).

10. Farshadfar, E., M. Mohammadi, M. Aghaee and Z. Vaisi. 2012. GGE biplot analysis of genotype $\times$ environment interaction in wheat-barley disomic addition lines. Australian Journal of Crop Science, 6(6): 1074-1079.

11. Gangadhar, K., S.A. Biradar, B.K. Desi, K. Ajithkumar and R. Rajanna. 2018. Growth and quality parameter of safflower as influenced by different row proportion in intercropping system of safflower (Carthamus tincorius L.) and linseed (Linum usitatissimum L.) under Rainfed condition. Journal of Pharmacognosy and Phytochemistry, 7(2): 1549-1554.

12. Gebremedhin, W., M. Firew and B. Tesfye. 2014. Stability analysis of food barley genotypes in northern Ethiopia. African Crop Science Journal, 22(2): 145-153.

13. Gollob, H.F. 1968. A Statistical Model Which Combines Features of Factor Analytic and Analysis of Variance Techniques. Psychometrika, 33: 73-115.

14. Kaiser, D.E., J.J. Wiersma and J.A. Anderson. 2014. Genotype and environment variation in elemental composition of spring wheat flag leaves. Agronomy Journal, 106: 324-336.

15. Karimizadeh, R., H. Dehghani and Z. Dehghanpour. 2008. Use of AMMI method for estimating genotype $\times$ environment interaction in early maturing corn hybrids. Seed Plant Improvement Journal, 23(4): $531-546$ (In Persian).

16. Kendal, E. 2016. GGE biplot analysis of multi-environment yield trials in barley (Hordeum vulgare L.) cultivars. Ekin Journal, 2(1): 90-99. 
17. Khomari, A., Kh. Mostafsvi and A. Mohammadi. 2017. Stability Study of Yield in Sunflower (Helianthus annuus L.) Cultivars using AMMI Method. Journal of Crop Breeding, 9(23): 117- 124 (In Persian).

18. Khomari, A., Kh. Mostafsvi and A. Mohammadi. 2018. Evaluation of yield stability of winter barley varieties (Hordeum vulgare L.) using additive main effects and multiplicative interaction method. Journal of crop production, 11(2): 185- 195 (In Persian). http://dx.doi.org/10.22069/ejcp. 2018.13567.2043

19. Liu, L., L.L. Guan and Y.X. Yang. 2016. A review of fatty acids and genetic characterization of safflower (Carthamus tinctorius L.) seed oil. World journal tradit chin med, 2(2): 48-52.

20. Ma'ali, S.H. 2008. Additive main effects and multiplicative interaction analysis of maize yield trials in South Africa. S. Afr. J. Plant Soil, 25: 185-193.

21. Makumbi, D., A. Diallo, K. Kanampiu, S. Mugo and H. Karaya. 2015. Agronomic performance and genotype $x$ environment interaction of herbicide-resistant maize varieties in Eastern Africa. Crop Science, 55: 540-555.

22. Moghaddam, M.J. and S.S. Pourdad. 2009. Comparison of parametric and nonparametric methods for analysing genotype $\times$ environment interactions in Safflower (Carthamus tinctorius L.). Journal of Agricultural Science, Cambridge. 147: 601-612.

23. Mohammadi, R. and A. Amri. 2011. Cultivar x environment interaction for duram wheat grain yield and selection for drought tolerance in irrigated and droughted environments. J. Crop Sci. Biotech., 14: $265-274$.

24. Mohammadi, R., M. Armion, H. Esmail Zad, M.M. Ahmadi and D. Sadegh Zadeh Ahari. 2012. Genotype $\times$ Environment interaction for grain yield of rainfed durum wheat using the GGE bipot model. Seed and plant improvement journal, 3(28): 503-518 (In Persian).

25. Moreno-Gonzalez, J., J. Crossa and P.L. Cornelius. 2004. Genotype x environment interaction in multienvironment trials using shrinkage factors for AMMI models. Euphytica, 137: 119-127.

26. Mostafavi, Kh., H. Shojaei, M. Khodarahmi and A. Mohammadi. 2010. The interaction of genotype and environment in canola with using GGE biplot graphical methods, Third International Seminar of oilseeds and edible oils, Tehran, Coordination Center of Science and Industry oilseeds.

27. Mündel, H.H. 2008. Major achievements in safflower breeding and future challenges. In: Proceedings of Products, VIIth International Safflower Conference, 3-6 November, Wagga Wagga, Australia.

28. Nassir, A.L. and O.J. Ariyo. 2011. Genotype x Environment interaction and yield-stability analysis of rice grown in tropical inland swamp. Not Bot Hort AgrobotCluj, 39(1): 220-225.

29. Omrani S, A.M. Naji and M. Esmaeil Zadeh Moghadam. 2018. Evaluation of Yield Stability of Bread wheat (Triticum aestivum L.) Genotypes using Additive Main Effects and Multiplicative Interaction (AMMI). Journal of Crop Breeding, 10(25): 73-80 (In Persian).

30. Omrani, S., A.M. Naji and M. Esmaeil Zadeh Moghadam. 2017. Yield stability analysis of promising bread wheat lines in southern warm and dry agro climatic zone of Iran using GGE biplot model. Journal of Crop Breeding, 9(23): 157-165 (In Persian).

31. Purchase, J.L., H. Hatting and C.S. Van-Deventer. 2000. Cultivar x environment interaction of winter wheat (Triticum aestivum L.) in South Africa: II Stability analysis of yield performance. S. Afr. J. Plant Soil, 17: 101107.

32. Sadeghi, S.M., H. Samizadeh, E. Amiri and M. Ashouri. 2011. Additive main effect sand multiplicative interactions (AMMI) analysis of dry leaf yield in tobacco hybrids across environments. African Journal of Biotechnology, 10: 4358-4364.

33. Sujatha, M. 2008. Biotechnological interventions for genetic improvement of safflower. In: Proceedings of VIIth International Safflower Conference, 3-6 November, Wagga Wagga, Australia.

34. Uher, J. 2008. Safflower in European Floriculture. In: Proceeding of VIIth International Safflower Conference, 3-6 November, Wagga Wagga, Australia.

35. Vaezi, B., J. Ahmadi and H. Naraki. 2011. Genotype $\times$ environment interaction and stability analysis for safflower (Carthamus tinctorius L.) genotypes under warm rainfed conditions. Iranian Journal of Crop Science. 13: 395-407 (In Persian).

36. Yang, R., J. Crossa, P. Cornelius and J. Bugueno. 2009. Biplot analysis of genotype x environment interaction: Proceed with caution. Crop Science, 49: 1564-1576. 


\title{
Application of multivariable of Additive Main Effects and Multiplicative Interaction and Biplot Graphical Analysis Multivariate Methods on the Study of Genotype- Environment Interaction on Safflower Genotypes Grain Yield
}

\author{
Saeed Omrani ${ }^{1}$, Ali Omrani ${ }^{2}$, Mahvash Afshari ${ }^{3}$, Ali Saremi-rad ${ }^{4}$, Siavash Bardehji $^{3}$ and \\ Peyman Foroozesh \\ 1- Ph.D. Student, Department of Agronomy and Plant Breeding, Isfahan University of Technology, Isfahan, Iran \\ (Corresponding author: s.omrani70@gmail.com) \\ 2- Crop and Horticultural Science Research Department, Ardabil (Moghan) Agricultural and Natural Resources Research \\ and Education Center, Agricultural Research, Education and Extension Organization (AREEO), Moghan, Iran \\ 3- Ph.D. Student, Department of Agronomy and Plant Breeding, Isfahan University of Technology, Isfahan, Iran \\ 4 and 5- Ph.D. Student and Assistant Professor, Department of Agronomy and Plant Breeding, Karaj Branch, Islamic \\ Azad Universitv. Karaj, Iran \\ Received: March 18, $2019 \quad$ Accepted: October 31, 2019
}

\begin{abstract}
To evaluate the effect of different environments on grain yield, 25 genotypes of safflower along with one control group namely Kouse were studied in three locations (Tehran, Birjand and Isfahan) and three years (from 2015 to 2017), in each using a completely randomized block design with three replications. The results obtained from AMMI analysis demonstrated that the main effects of genotype, environment and genotype $\times$ environment interaction were highly significant. The first four principal components justified around $83 \%$ of the sum of squares of the interactions, and explained $40 \%, 19 \%, 13 \%$ and $9 \%$ of variances, respectively. Drawing the biplot of the first principal component and the average yield for genotypes and environments suggested that genotypes including Kouse, E2417, PI-250537 with higher than average yields and desired stability were selected. Biplot of the first two principal components showed that the interaction between genotypes Kouse, Hartman and PI-250537 were identified as genotypes with good compatibility. Based on GGE Biplot method, two main components of the spatial regression models explained about $96 \%$ of grain yield variability. Biplot of relevance between environments confirmed the existence of almost identical reaction between genotypes in both locations including Isfahan and Tehran, but genotypes in Birjand showed a different reaction than these two environments. Based on the polygonal figure, genotypes including A2 in Birjand and Hartman in Isfahan as well as Tehran had a higher degree of narrative compatibility and performance stability. Genotype of Hartman and Isfahan location were the most ideal genotype and environments compared to other genotypes and environments.
\end{abstract}

Keywords: AMMI, GGE Biplot, Mega-Environments, Stability Analysis, Safflower 